ARTICLE

Received 22 May 2014 | Accepted 4 Sep 2014 | Published 8 Oct 2014

DOl: $10.1038 /$ ncomms6156

OPEN

\title{
Suppressing qubit dephasing using real-time Hamiltonian estimation
}

\author{
M.D. Shulman ${ }^{1, \star}$, S.P. Harvey ${ }^{1, \star}$, J.M. Nichol ${ }^{1, \star}$, S.D. Bartlett ${ }^{2}$, A.C. Doherty ${ }^{2}$, V. Umansky ${ }^{3}$ \& A. Yacoby ${ }^{1}$
}

Unwanted interaction between a quantum system and its fluctuating environment leads to decoherence and is the primary obstacle to establishing a scalable quantum information processing architecture. Strategies such as environmental and materials engineering, quantum error correction and dynamical decoupling can mitigate decoherence, but generally increase experimental complexity. Here we improve coherence in a qubit using real-time Hamiltonian parameter estimation. Using a rapidly converging Bayesian approach, we precisely measure the splitting in a singlet-triplet spin qubit faster than the surrounding nuclear bath fluctuates. We continuously adjust qubit control parameters based on this information, thereby improving the inhomogenously broadened coherence time $\left(T_{2}^{*}\right)$ from tens of nanoseconds to $>2 \mu \mathrm{s}$. Because the technique demonstrated here is compatible with arbitrary qubit operations, it is a natural complement to quantum error correction and can be used to improve the performance of a wide variety of qubits in both meteorological and quantum information processing applications.

\footnotetext{
${ }^{1}$ Department of Physics, Harvard University, Cambridge, Massachusetts 02138, USA. ${ }^{2}$ Centre for Engineered Quantum Systems, School of Physics, The University of Sydney, Sydney, New South Wales 2006, Australia. ${ }^{3}$ Braun Center for Submicron Research, Department of Condensed Matter Physics, Weizmann Institute of Science, Rehovot 76100, Israel. * These authors contributed equally to this work. Correspondence and requests for materials should be addressed to A.Y. (email: yacoby@physics.harvard.edu).
} 
$\mathrm{H}$ amiltonian parameter estimation is a rich field of active experimental and theoretical research that enables precise characterization and control of quantum systems ${ }^{1}$. For example, magnetometry schemes employing Hamiltonian learning have demonstrated dynamic range and sensitivities exceeding those of standard methods ${ }^{2,3}$. Such applications focused on estimating parameters that are quasistatic on experimental timescales. However, the effectiveness of Hamiltonian learning also offers exciting prospects for estimating fluctuating parameters responsible for decoherence in quantum systems.

The quantum system that we study is a singlet-triplet $\left(S-T_{0}\right)$ qubit $^{4,5}$ which is formed by two gate-defined lateral quantum dots (QDs) in a GaAs/AlGaAs heterostructure (Fig. 1a, Supplementary Fig. 1), similar to that of refs 6,7. The qubit can be rapidly initialized in the singlet state $|S\rangle$ in $\approx 20$ ns and read out with $98 \%$ fidelity in $\approx 1 \mu$ s (refs 8,9; Supplementary Fig. 2). Universal quantum control is provided by two distinct drives ${ }^{10}$ : the exchange splitting, $J$, between $|S\rangle$ and $\left|T_{0}\right\rangle$, and the magnetic field gradient, $\Delta B_{z}$, due to the hyperfine interaction with host $\mathrm{Ga}$ and As nuclei. The Bloch sphere representation for this qubit can be seen in Fig. 1b. In this work, we focus on qubit evolution

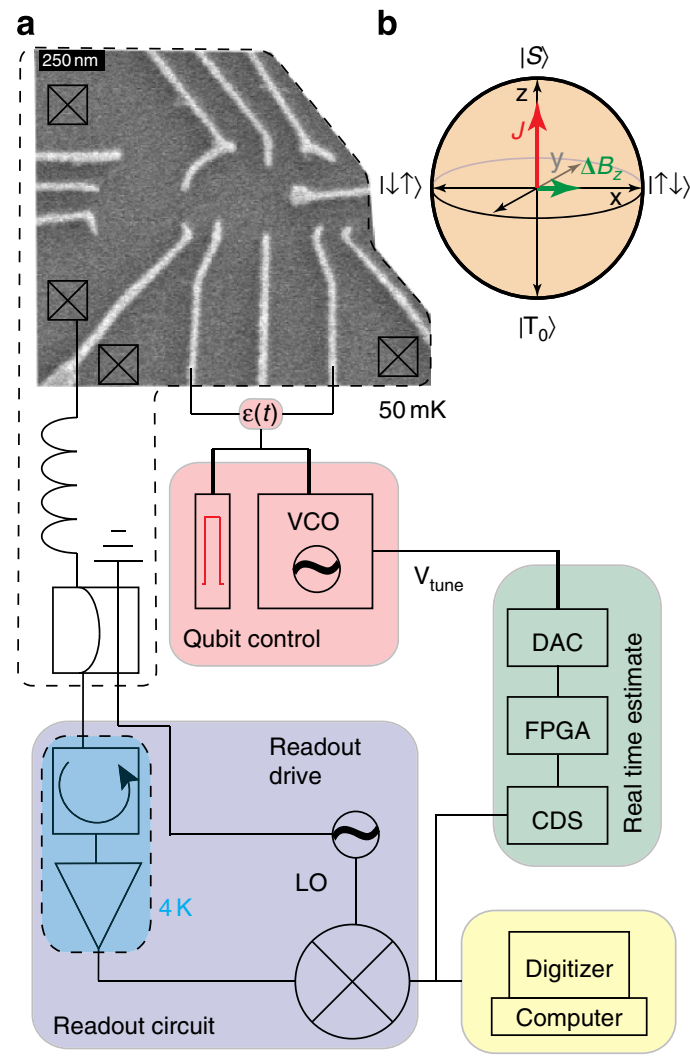

Figure 1 | Experimental apparatus. (a) A scanning electron microscope image of the double QD with a schematic of the apparatus used for adaptive qubit control. A floating metal gate protruding from the right can be seen which increases the capacitance between the qubit and an adjacent qubit (not pictured), which is left inactive for this work. The reflected read-out drive signal is demodulated to $D C$, digitized by a correlated double sampler (CDS), and $\Delta B_{z}$ is estimated in real time by the field-programmable gate array (FPGA). The FPGA updates the digital to analogue converter (DAC) to keep the voltage controlled oscillator (VCO) resonant with the estimated value of $\Delta B_{z}$. The VCO controls the voltage detuning, $\epsilon(t)$ between the QDs, which, in turn, modulates $J$ at $\Omega$. (b) The Bloch sphere representation for the $S-T_{0}$ qubit showing the two axes of control, $J$ and $\Delta B_{z}$. around $\Delta B_{z}$ (Fig. 2a). Due to statistical fluctuations of the nuclei, $\Delta B_{z}$ varies randomly in time, and consequently oscillations around this field gradient decay in a time $T_{2}^{*} \approx 10 \mathrm{~ns}$ (ref. 4). A nuclear feedback scheme relying on dynamic nuclear polarization $^{11}$ can be employed to set the mean gradient, $\left(g^{\star} \mu_{\mathrm{B}} \Delta B_{z} / h \approx 60 \mathrm{MHz}\right.$ in this work) as well as reduce the variance of the fluctuations. Here, $g^{\star} \approx-0.44$ is the effective gyromagnetic ratio in GaAs, $\mu_{\mathrm{B}}$ is the Bohr magneton and $h$ is Planck's constant. In what follows, we adopt units where $g^{\star} \mu_{\mathrm{B}} /$ $h=1$. The nuclear feedback relies on the avoided crossing between the $|S\rangle$ and $\left|T_{+}\right\rangle$states. When the electrons are brought adiabatically through this crossing, their total spin changes by $\Delta m_{\mathrm{s}}= \pm 1$, which is accompanied by a nuclear spin flip to conserve angular momentum. With the use of this feedback, the coherence time improves to $T_{2}^{*} \approx 100$ ns (ref. 11 ; Fig. 2b), limited by the low nuclear pumping efficiency ${ }^{10}$. Crucially, the residual fluctuations are considerably slower than the timescale of qubit operations ${ }^{12}$.

In this work we employ techniques from Hamiltonian estimation to prolong the coherence of a qubit by more than a factor of 30. Importantly, our estimation protocol, which is based on recent theoretical work ${ }^{13}$, requires relatively few measurements $(\approx 100)$ which we perform rapidly enough (total time $\approx 100 \mu \mathrm{s}$ ) to resolve the qubit splitting faster than its characteristic fluctuation time. We adopt a paradigm in which we separate experiments into 'estimation' and 'operation' segments, and we use information from the former to optimize control parameters for the latter in real-time. Our method dramatically prolongs coherence without using complex pulse sequences such as those required for non-identity dynamically decoupled operations $^{14}$.

\section{Results}

Rotating frame $S-T_{0}$ qubit. To take advantage of the slow nuclear dynamics, we introduce a method that measures the fluctuations and manipulates the qubit on the basis of precise knowledge but not precise control of the environment. We operate the qubit in the rotating frame of $\Delta B_{z}$, where qubit rotations are driven by modulating $J$ at the frequency $\frac{\Omega_{J}}{2 \pi}=\Delta B_{z}$ (refs 15,16). This is in contrast to traditional modes of operation of the $S-T_{0}$ qubit, which rely on DC voltage pulses. To measure Rabi oscillations, the qubit is adiabatically prepared in the ground state of $\Delta B_{z}(|\psi\rangle=|\uparrow \downarrow\rangle)$, and an oscillating $J$ is switched on (Fig. 2e), causing the qubit to precess around $J$ in the rotating frame. In addition, we perform a Ramsey experiment (Fig. 2c) to determine $T_{2}^{*}$, and as expected, we observe the same decay (Fig. 2d) as Fig. 2b. More precisely, the data in Fig. 2d represent the average of 1,024 experimental repetitions of the same qubit operation sequence immediately following nuclear feedback. The feedback cycle resets $\Delta B_{z}$ to its mean value $(60 \mathrm{MHz})$ with residual fluctuations of $\left(\sqrt{2} \pi T_{2}^{*}\right)^{-1} \approx 10 \mathrm{MHz}$ between experimental repetitions. However, within a given experimental repetition, $\Delta B_{z}$ is approximately constant. Therefore we present an adaptive control scheme where, following nuclear feedback, we quickly estimate $\Delta B_{z}$ and tune $\frac{\Omega_{J}}{2 \pi}=\Delta B_{z}$ to prolong qubit coherence (Fig. 3a).

Bayesian estimation. To estimate $\Delta B_{z}$, we repeatedly perform a series of single-shot measurements after allowing the qubit to evolve around $\Delta B_{z}$ (using DC pulses) for some amount of time (Fig. 2a). Rather than fixing this evolution time to be constant for all trials, we make use of recent theoretical results in Hamiltonian parameter estimation ${ }^{13,16,17}$ and choose linearly increasing evolution times, $t_{k}=k t_{\text {samp}}$, where $k=1,2, \ldots N$. We choose the sampling time $t_{\text {samp }}$ such that the estimation bandwidth $\mathcal{B}=\frac{1}{2 t_{\text {samp }}}$ 
a

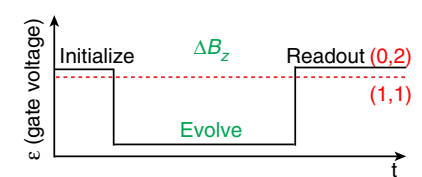

b

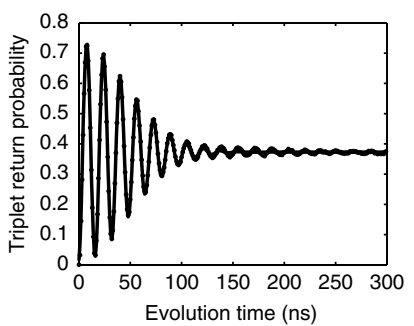

C

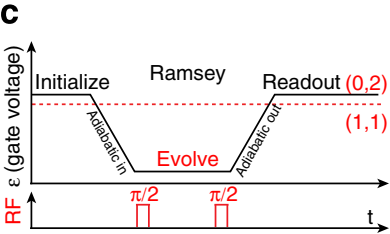

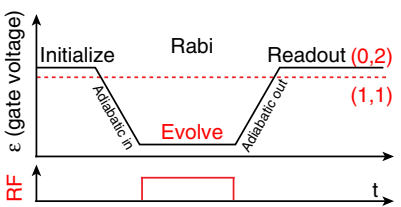

d

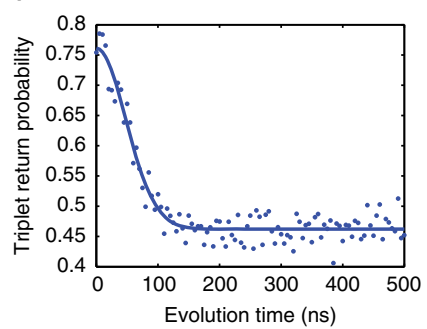

f

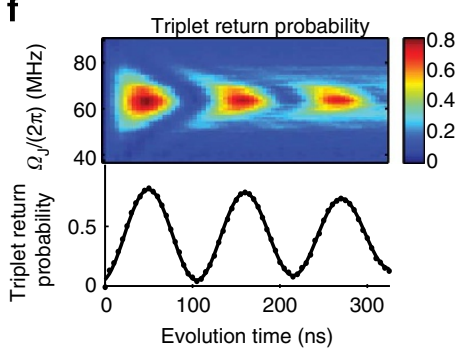

Figure $2 \mid \Delta \boldsymbol{B}_{\mathbf{z}}$ oscillations. (a) The pulse sequence used to estimate $\Delta B_{z}$. (b) Using nuclear feedback, $\Delta B_{z}$ oscillations decay in a coherence time $T_{2}^{*} \approx 66 \mathrm{~ns}$ due to residual slow fluctuations in $\Delta B_{z}$. (c) The Ramsey sequence used to operate the $S-T_{0}$ qubit in the rotating frame. (d) The Ramsey contrast (blue dots) decays in a characteristic time (solid line fit $T_{2}^{*} \approx 68 \mathrm{~ns}$ ) similar to the oscillations in $\mathbf{b}$ due to the same residual slow fluctuations in $\Delta B_{z}$. (e) The Rabi pulse sequence used to drive the qubit in the rotating frame. (f) The rotating frame $S-T_{0}$ qubit exhibits the typical behaviour when sweeping drive frequency and time (top). When driven on resonance (bottom), the qubit undergoes Rabi oscillations, demonstrating control in the rotating frame.

a

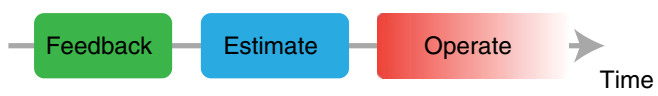

b

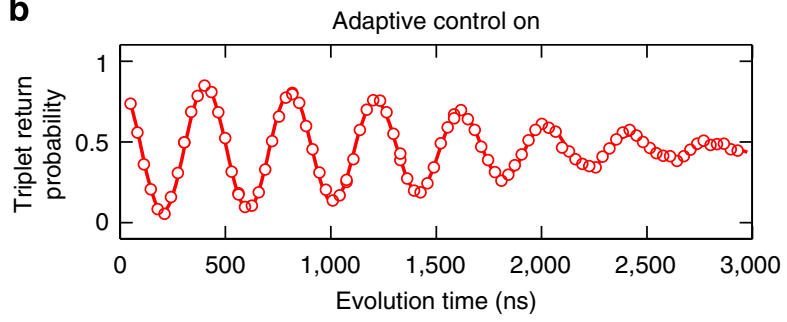

C

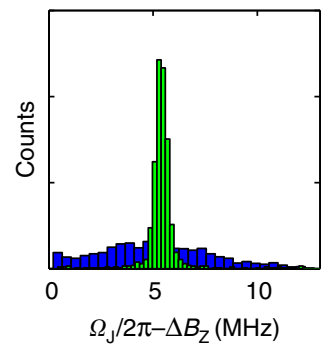

d

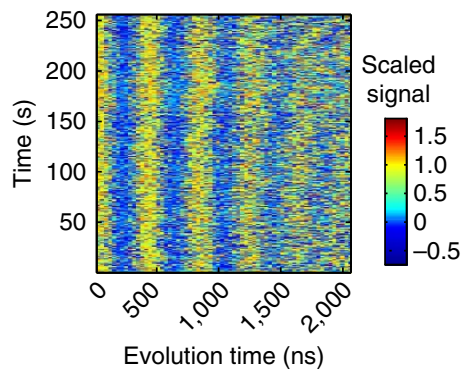

Figure 3 | Adaptive control. (a) For these measurements we first perform our standard nuclear feedback, then quickly estimate $\Delta B_{z}$ and update the qubit control, then operate the qubit at the correct driving frequency. (b) Using adaptive control, we perform a Ramsey experiment (deliberately detuned to see oscillations) and obtain coherence times of $T_{2}^{*} \approx 2,066$ ns. (c) Histograms of measured Ramsey detunings with (green) and without (blue) adaptive control. For clarity, these data were taken with a different mean detuning than those in $\mathbf{b}$. (d) Raw data for 1,024 consecutive Ramsey experiments with adaptive control lasting $250 \mathrm{~s}$ in total. A value of 1 corresponds to $\left|T_{0}\right\rangle$ and 0 corresponds to $|S\rangle$. Stabilized oscillations are clearly visible in the data, demonstrating the effect of adaptive control. is several times larger than the magnitude of the residual fluctuations in $\Delta B_{z}$, roughly $10 \mathrm{MHz}$. With a Bayesian approach to estimate $\Delta B_{z}$ in real-time, the longer evolution times (large $k$ ) leverage the increased precision obtained from earlier measurements to provide improved sensitivity, allowing the estimate to outperform the standard limit associated with repeating measurements at a single evolution time. Denoting the outcome of the $k$ th measurement as $m_{k}$ (either $|S\rangle$ or $\left|T_{0}\right\rangle$ ), we define $P\left(m_{k} \mid \Delta B_{z}\right)$ as the conditional probability for $m_{k}$ given a value $\Delta B_{z}$. We write

$$
P\left(m_{k} \mid \Delta B_{z}\right)=\frac{1}{2}\left[1+r_{k}\left(\alpha+\beta \cos \left(2 \pi \Delta B_{z} t_{k}\right)\right)\right],
$$

where $r_{k}=1(-1)$ for $m_{k}=|S\rangle\left(\left|T_{0}\right\rangle\right)$, and $\alpha=0.25$ and $\beta=0.67$ are parameters determined by the measurement error and axis of rotation on the Bloch sphere (see Methods). Since we assume that earlier measurement outcomes do not affect later ones (that is, that there is no measurement back-action), we write the conditional probability for $\Delta B_{z}$ given the results of $N$ measurements as:

$$
\begin{gathered}
P\left(\Delta B_{z} \mid m_{N}, m_{N-1}, \ldots m_{1}\right) \\
=P\left(\Delta B_{z} \mid m_{N-1}, \ldots m_{1}\right) \cdot P\left(\Delta B_{z} \mid m_{N}\right) \\
=\prod_{k=1}^{N} P\left(\Delta B_{z} \mid m_{k}\right) .
\end{gathered}
$$

Using Bayes' rule, that is, $P\left(\Delta B_{z} \mid m_{k}\right)=P\left(m_{k} \mid \Delta B_{z}\right) P\left(\Delta B_{z}\right) / P\left(m_{k}\right)$, and equation (1), we can rewrite equation (3) as:

$$
P\left(\Delta B_{z} \mid m_{N}, m_{N-1}, \ldots m_{1}\right)=P_{0}\left(\Delta B_{z}\right) \mathcal{N} \prod_{k=1}^{N}\left(1+r_{k}\left(\alpha+\beta \cos \left(2 \pi \Delta B_{z} t_{k}\right)\right)\right],
$$

where $\mathcal{N}$ is a normalization constant and $P_{0}\left(\Delta B_{z}\right)$ is a prior distribution to which the algorithm is empirically insensitive, and which we take to be a constant over the estimation bandwidth. After the last measurement, we find the value of $\Delta B_{z}$ that maximizes the posterior distribution $P\left(\Delta B_{z} \mid m_{N}, m_{N-1}, \ldots m_{1}\right)$. 
Adaptive control. We implement this algorithm in real-time on a field-programmable gate array (FPGA), computing $P\left(\Delta B_{z}\right)$ for 256 values of $\Delta B_{z}$ between 50 and $70 \mathrm{MHz}$. With each measurement $m_{k}$, the read-out signal is digitized and passed to the FPGA, which computes $P\left(\Delta B_{z}\right)$ and updates an analogue voltage that tunes the frequency of a voltage controlled oscillator (Fig. 1a; Supplementary Note 1, Supplementary Fig. 3). Following the Nth sample, $\frac{\Omega_{J}}{2 \pi}$ nearly matches $\Delta B_{z}$, and since the nuclear dynamics are slow, the qubit can be operated with long coherence without any additional complexity. To quantify how well the FPGA estimate matches $\Delta B_{z}$, we perform a Ramsey experiment (deliberately detuned to observe oscillations) with this real-time tracking of $\Delta B_{z}$ and find optimal performance for $N \approx 120$, with a maximum experimental repetition rate, limited by the FPGA, of $250 \mathrm{kHz}$ and a sampling time $t_{\text {samp }}=12 \mathrm{~ns}$. Under these conditions, and making a new estimate after every 42 Ramsey experiments, we observe $T_{2}^{*} \approx 2,066 \mathrm{~ns}$, a 30 -fold increase in coherence (Fig. 3b). We note that these data are taken with the same pulse sequence as those in Fig. 2d. To further compare qubit operations with and without this technique, we measure Ramsey fringes for $\approx 250 \mathrm{~s}$ (Fig. 3d), and histogram the observed Ramsey detunings. With adaptive control we observe a stark narrowing of the observed frequency distribution, consistent with this improved coherence (Fig. 3c).

\section{Discussion}

Although the estimation scheme employed here is theoretically predicted to improve monotonically with $N$ (ref. 13), we find that there is an optimum $(N \approx 120)$, after which $T_{2}^{*}$ slowly decreases with increasing $N$ (Fig. 4a). A possible explanation for this trend is fluctuation of the nuclear gradient during the estimation period. To investigate this, we obtain time records of $\Delta B_{z}$ using the Bayesian estimate and find that its variance increases linearly in time at the rate of $(6.7 \pm 0.7 \mathrm{kHz})^{2} \mu \mathrm{s}^{-1}$ (Fig. $4 \mathrm{c}$ ). The observed linear behaviour suggests a model where the nuclear gradient diffuses, which can arise, for example, from dipolar coupling between adjacent nuclei. Using the measured diffusion of $\Delta B_{z}$, we simulate the performance of the Bayesian estimate as a function of $N$ (see Methods). Given that the simulation has no free parameters, we find good agreement with the observed $T_{2}^{*}$, indicating that indeed, diffusion limits the accuracy with which we can measure $\Delta B_{z}$ (Fig. $4 \mathrm{a}$ ).

This model suggests that increasing the rate of measurements during estimation will improve the accuracy of the Bayesian estimate. Because our FPGA limits the repetition rate of qubit operations to $250 \mathrm{kHz}$, we demonstrate the effect of faster measurements through software post-processing with the same Bayesian estimate. To do so, we first use the same estimation sequence, but for the operation segment, we measure the outcome after evolving around $\Delta B_{z}$ for a single evolution time, $t_{\mathrm{evo}}$, rather than performing a rotating frame Ramsey experiment, and we repeat this experiment a total of $N_{\text {tot }}$ times. In processing, we perform the Bayesian estimate of each $\Delta B_{z, i}$, sort the data by adjusted time $\tau_{i}=\frac{\Delta B_{z, i} t_{\mathrm{evo}, i}}{\left\langle\Delta B_{z}\right\rangle}$ (for $i=1,2, \ldots N_{\mathrm{tot}}$ ), and average together points of similar $\tau$ to observe oscillations (see Methods). We fit the decay of these oscillations to extract $T_{2}^{*}$ and the precision of the Bayesian estimate, $\sigma_{\Delta B_{z}}=\left(\sqrt{2} \pi T_{2}^{*}\right)^{-1}$. For the same operation and estimation parameters, we find that $T_{2}^{*}$ extracted from software post-processing agrees with that extracted from adaptive control Supplementary Fig. 4, Supplementary Note 2. Using a repetition rate as high as $667 \mathrm{kHz}$, we show coherence times above 2,800 ns, corresponding to an error of $\sigma_{\Delta B z}=80 \mathrm{kHz}$ (Fig. $4 \mathrm{~d}$ ), indicating that improvements are easily attainable by using faster (commercially available) FPGAs.

In addition, we use this post-processing to examine the effect of this technique on the duty cycle of experiments as well as the stability of the $\Delta B_{z}$ estimate. To do so we introduce a delay $T_{\text {delay }}$ between the estimation of $\Delta B_{z}$ and the single evolution measurement performed in place of the operation. We a
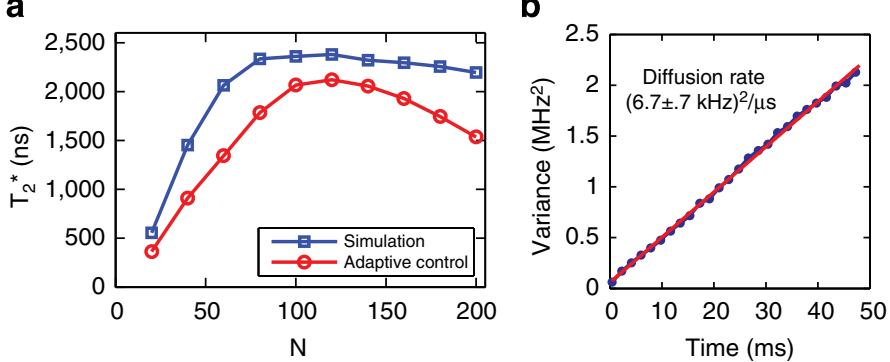

C

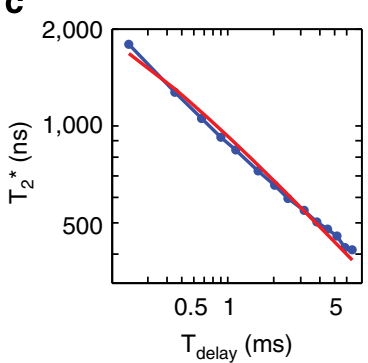

d

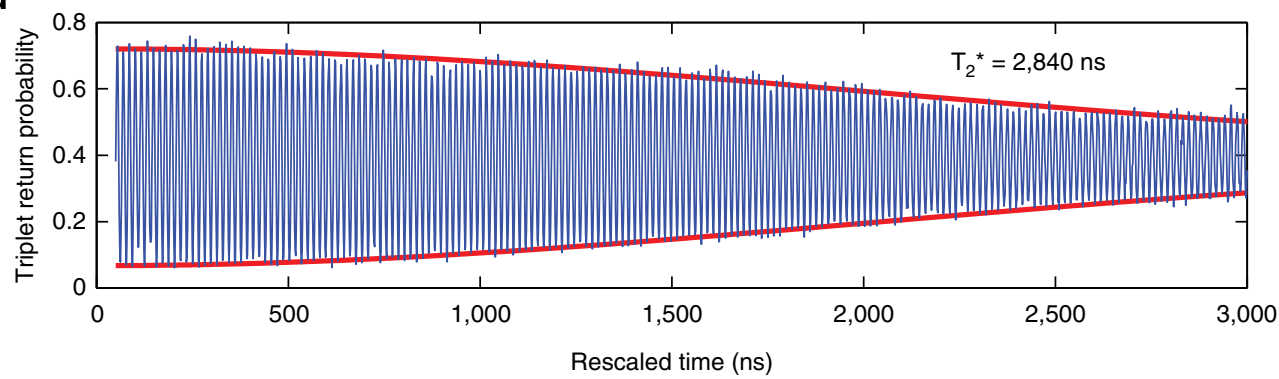

Figure $4 \mid \Delta \mathbf{B}_{\mathbf{z}}$ diffusion. (a) The coherence time, $T_{2}^{*}$ using the adaptive control and for a simulation show a peak, indicating that there is an optimal number of measurements to make when estimating $\Delta B_{z}$. (b) When many time traces of $\Delta B_{z}$ are considered, their variance grows linearly with time, indicating a diffusion process. (c) The scaling of $T_{2}^{*}$ as a function of $T_{\text {delay }}$ for software scaled data is consistent with diffusion of $\Delta B_{z}$. The red line is a fit to a diffusion model. (d) The performance of the Bayesian estimate of $\Delta B_{z}$ can be estimated using software post-processing, giving $T_{2}^{*} \approx 2,840 \mathrm{~ns}$, which corresponds to a precision of $\sigma_{\Delta B_{z}}=80 \mathrm{kHz}$. 
find $T_{2}^{*}=\left(a+b T_{\text {delay }}^{c}\right)^{-1 / 2}$, where $c=0.99$ (Fig. $4 c$ ), consistent with diffusion of $\Delta B_{z}$. Indeed, this dependence underscores the potential of adaptive control, since it demonstrates that after a single estimation sequence, the qubit can be operated for $>1 \mathrm{~ms}$ with $T_{2}^{*}>1 \mu \mathrm{s}$. Thus, adaptive control need not significantly reduce the experimental duty cycle.

In this work, we have used real-time adaptive control on the basis of Hamiltonian parameter estimation of a $S-T_{0}$ spin qubit to prolong $T_{2}^{*}$ from 70 ns to $>2 \mu$ s. Dephasing due to nuclear spins has long been considered a significant obstacle to quantum information processing using semiconductor spin qubits ${ }^{18}$, and elimination of nuclear spins is an active and fruitful area of research ${ }^{19-21}$. However, here we have shown that with a combination of nuclear feedback, rotating frame $S-T_{0}$ spin resonance, and real-time Hamiltonian estimation, we are able to achieve ratios of coherence times to operation times in excess of 200 without recourse to dynamical decoupling ${ }^{12,22,23}$. If the same adaptive control techniques were applied to gradients as high as $1 \mathrm{GHz}$ (ref. 10), ratios exceeding 4,000 would be possible, and longer coherence times may be attainable with more sophisticated techniques ${ }^{13}$. Though the observed coherence times are still smaller than the Hahn echo time, $T_{2}^{\text {echo }}$ (ref. 12), the method we have presented is straightforward to implement, compatible with arbitrary qubit operations, and general to all qubits that suffer from non-Markovian noise. Looking ahead, it is likely, therefore, to have a key role in realistic quantum error correction efforts $^{24-27}$, where even modest improvements in baseline error rate greatly diminish experimental complexity and enhance prospects for a scalable quantum information processing architecture.

\section{Methods}

Bayesian estimate. We wish to calculate the probability that the nuclear magnetic field gradient has a certain value, $\Delta B_{z}$, given a particular measurement record comprising $N$ measurements. We follow the technique in Sergeevich et al. ${ }^{13}$ with slight modifications. Writing the outcome of the $k$ th measurement as $m_{k}$, we write this probability distribution as

$$
P\left(\Delta B_{z} \mid m_{N}, m_{N-1}, \ldots m_{1}\right) .
$$

To arrive at an expression for this distribution, we will write down a model for the dynamics of the system, that is, $P\left(m_{N}, m_{N-1}, \ldots m_{1} \mid \Delta B_{z}\right)$. Using Bayes' rule we can relate the two equations as

$$
\begin{aligned}
& P\left(\Delta B_{z} \mid m_{N}, m_{N-1}, \ldots m_{1}\right) \cdot P\left(m_{N}, m_{N-1}, \ldots m_{1}\right) \\
& \quad=P\left(m_{N}, m_{N-1}, \ldots m_{1} \mid \Delta B_{z}\right) \cdot P\left(\Delta B_{z}\right) .
\end{aligned}
$$

First, we seek a model that can quantify $P\left(m_{N}, m_{N-1}, \ldots m_{1} \mid \Delta B_{z}\right)$ that accounts for realistic errors in the system, namely measurement error, imperfect state preparation and error in the axis of rotation around the Bloch sphere. For simplicity, we begin with a model that accounts only for measurement error. Denoting the error associated with measuring a $|S\rangle\left(\left|T_{0}\right\rangle\right)$ as $\eta_{\mathrm{S}}\left(\eta_{\mathrm{T}}\right)$, we write

$$
\begin{aligned}
& P\left(S \mid \Delta B_{z}\right)=\left(1-\eta_{\mathrm{S}}\right) \cos ^{2}\left(2 \pi \Delta B_{z} t_{k} / 2\right)+\eta_{\mathrm{T}} \sin ^{2}\left(2 \pi \Delta B_{z} t_{k} / 2\right) \\
& P\left(T_{0} \mid \Delta B_{z}\right)=\left(1-\eta_{\mathrm{T}}\right) \sin ^{2}\left(2 \pi \Delta B_{z} t_{k} / 2\right)+\eta_{\mathrm{S}} \cos ^{2}\left(2 \pi \Delta B_{z} t_{k} / 2\right)
\end{aligned}
$$

We combine these two equations and write

$$
P\left(m_{k} \mid \Delta B_{z}\right)=\frac{1}{2}\left[1+r_{k}\left(\alpha+\beta \cos \left(2 \pi \Delta B_{z} t_{k}\right)\right)\right]
$$

where $r_{k}=1(-1)$ for $m_{k}=|S\rangle\left(\left|T_{0}\right\rangle\right)$ and $\alpha$ and $\beta$ are given by

$$
\alpha=\left(\eta_{\mathrm{T}}-\eta_{\mathrm{S}}\right), \quad \beta=\left(1-\eta_{\mathrm{S}}-\eta_{\mathrm{T}}\right) .
$$

Next, we generalize the model to include the effects of imperfect state preparation, and the presence of non-zero $J$ during evolution, which renders the initial state non-orthogonal to the axis of rotation around the Bloch sphere (see above). We assume that the angle of rotation around the Bloch sphere lies somewhere in the $x-z$ plane and makes an angle $\theta$ with the $z$ axis. We define $\delta=\cos ^{2}(\theta)$. Next, we include imperfect state preparation by writing the density matrix $\left.\rho_{\text {init }}=(1-\epsilon)|S\rangle\langle S|\right)+\epsilon\left|T_{0}\right\rangle\left\langle T_{0}\right|$. With this in hand, we can write down the model

$$
P\left(S \mid \Delta B_{z}\right)=\eta_{\mathrm{T}}+\frac{1}{2}\left(1-\eta_{\mathrm{S}}-\eta_{\mathrm{T}}\right)\left\{1+(1-2 \epsilon)\left[\delta+(1-\delta) \cos \left(2 \pi \Delta B_{z} t_{k}\right)\right]\right\},
$$

$$
P\left(T_{0} \mid \Delta B_{z}\right)=\eta_{\mathrm{S}}+\frac{1}{2}\left(1-\eta_{\mathrm{S}}-\eta_{\mathrm{T}}\right)\left\{1-(1-2 \epsilon)\left[\delta+(1-\delta) \cos \left(2 \pi \Delta B_{z} t_{k}\right)\right]\right\} .
$$

Using the same notation for $r_{k}=1(-1)$ for $m_{k}=|S\rangle\left(\left|T_{0}\right\rangle\right)$, we rewrite this in one equation as

$$
P\left(m_{k} \mid \Delta B_{z}\right)=\frac{1}{2}\left[1+r_{k}\left(\alpha+\beta \cos \left(2 \pi \Delta B_{z} t_{k}\right)\right)\right],
$$

where we now have

$$
\begin{gathered}
\alpha=\eta_{\mathrm{T}}-\eta_{\mathrm{S}}+\left(1-\eta_{\mathrm{S}}-\eta_{\mathrm{T}}\right)(\delta-2 \epsilon \delta) \\
\beta=\left(1-\eta_{\mathrm{S}}-\eta_{\mathrm{T}}\right)(1-\delta)(1-2 \epsilon) .
\end{gathered}
$$

We find the best performance for $\alpha=0.25$ and $\beta=0.67$, which is consistent with known values for qubit errors.

We next turn our attention to implementing Bayes' rule to turn this model into a probability distribution for $\Delta B_{z}$. First, we assume that all measurements are statistically independent, allowing us to write

$$
\begin{aligned}
P\left(\Delta B_{z} \mid m_{N}, m_{N-1}, \ldots m_{1}\right) & =P\left(\Delta B_{z} \mid m_{N}\right) \cdot P\left(\Delta B_{z} \mid m_{N-1}, \ldots m_{1}\right) \\
& =\prod_{k=1}^{N} P\left(\Delta B_{z} \mid m_{k}\right) .
\end{aligned}
$$

We next use Bayes rule (6) and rewrite this equation as

$$
P\left(\Delta B_{z} \mid m_{N}, m_{N-1}, \ldots m_{1}\right)=\prod_{k=1}^{N} P\left(m_{k} \mid \Delta B_{z}\right) \frac{P\left(\Delta B_{z}\right)}{P\left(m_{k}\right)} .
$$

Using our model (13) we can rewrite this as

$$
P\left(\Delta B_{z} \mid m_{N}, m_{N-1}, \ldots m_{1}\right)=\mathcal{N} P_{0}\left(\Delta B_{z}\right) \prod_{k=1}^{N}\left[1+r_{k}\left(\alpha+\beta \cos \left(2 \pi \Delta B_{z} t_{k}\right)\right)\right],
$$

where $\mathcal{N}$ is a normalization constant, and $P_{0}\left(\Delta B_{z}\right)$ is a prior distribution for $\Delta B_{z}$ which we take to be a constant over the estimation bandwidth, and to which the estimator is empirically insensitive. With this formula, it is simple to see that the posterior distribution for $\Delta B_{z}$ can be updated in real time with each successive measurement. After the $N$ th measurement, we choose the value for $\Delta B_{z}$, which maximizes the posterior distribution (18).

Simulation with diffusion. We simulate the performance of our software scaling and hardware (FPGA) estimates of $\Delta B_{z}$ using the measured value of the diffusion rate. We assume that $\Delta B_{z}$ obeys a random walk, but assume that during a single evolution time $t_{k}, \Delta B_{z}$ is static. This assumption is valid when $\sqrt{t_{N} \mathcal{D}} T_{2}^{*} \ll 1$, where $\mathcal{D}$ is the diffusion rate of $\Delta B_{z}$. For an estimation of $\Delta B_{z}$ with $N$ different measurements, we generate a random walk of $N$ different values for $\Delta B_{z}$ (using the measured diffusion), simulate the outcome of each measurement, and compute the Bayesian estimate of $\Delta B_{z}$ using the simulated outcomes. By repeating this procedure 4,096 times, and using the mean squared error, MSE $=$ $\left\langle\left(\Delta B_{z}-\Delta B_{z}^{\text {estimated }}\right)^{2}\right\rangle$ as a metric for performance, we can find the optimal number of measurements to perform. To include the entire error budget of the FPGA apparatus, we add to this MSE the error from the phase noise of the VCO, the measured voltage noise on the analogue output controlling the VCO, and the diffusion of $\Delta B_{z}$ during the 'operation' period of the experiment.

Software post-processing. The estimate of $\Delta B_{z}$ can be independently verified using software analysis. In this experiment, we use the same method to estimate $\Delta B_{z}$ as in the adaptive control experiment, but in the operation segment perform oscillations around $\Delta B_{z}$ for verification. We choose $m$ different evolution times and measure each $n$ times for a total of $N_{\text {tot }}=m \times n$ measurements of $\Delta B_{z}$. In the $i$ th experiment $\left(i=1,2, \ldots N_{\mathrm{tot}}\right)$, we evolve for a time $t_{\mathrm{evo}, i}$, accumulating phase $\phi_{i}=$ $\Delta B_{z, i} t_{\mathrm{evo}, i}$. Because we make a precise measurement of $\Delta B_{z}$ at the start of each experiment, we can employ it to rescale the time, $t_{\mathrm{evo}, \text { i }}$, so that the phase accumulated for a given time is constant using the equation,

$$
\tau_{i} \equiv t_{\mathrm{evo}, i} \frac{\Delta B_{z}, i}{\left\langle\Delta B_{z}\right\rangle}
$$

This sets $\phi_{i}\left(\tau_{i}\right)=\left\langle\Delta B_{z}\right\rangle \tau_{i}$, with residual error arising from inaccuracy in the estimate of $\Delta B_{z i}$. The data are then sorted by $\tau$, and points of similar $\tau$ are averaged using a Gaussian window with $\sigma_{\tau}=0.5 \mathrm{~ns} \ll T \approx 16 \mathrm{~ns}$, where $\mathrm{T}$ is the period of the oscillations. 


\section{References}

1. Wiseman, Howard M. \& Milburn, Gerard J. Quantum Measurement and Control (Cambridge Univ. Press, 2010).

2. Waldherr, G. et al. High-dynamic-range magnetometry with a single nuclear spin in diamond. Nat. Nanotechnol. 7, 105-108 (2012).

3. Nusran, N. M., Ummal, M. \& Gurudev Dutt, M. V. High dynamic range magnetometry with a single electronic spin in diamond. Nat. Nanotechnol. 7, 109-113 (2012).

4. Petta, J. R. et al. Coherent manipulation of coupled electron spins in semiconductor quantum dots. Science 309, 2180-2184 (2005).

5. Maune, B. M. et al. Coherent singlet-triplet oscillations in a silicon-based double quantum dot. Nature 481, 344-347 (2011).

6. Dial, O. E. et al. Charge noise spectroscopy using coherent exchange oscillations in a singlet-triplet qubit. Phys. Rev. Lett. 110, 146804 (2013)

7. Shulman, M. D. et al. Demonstration of entanglement of electrostatically coupled singlet-triplet qubits. Science 336, 202-205 (2012).

8. Barthel, C., Reilly, D. J., Marcus, C. M., Hanson, M. P. \& Gossard., A. C. Rapid single-shot measurement of a singlet-triplet qubit. Phys. Rev. Lett. 103, 160503 (2009).

9. Reilly, D. J., Marcus, C. M., Hanson, M. P. \& Gossard, A. C. Fast single-charge sensing with a RF quantum point contact. Appl. Phys. Lett. 91, 162101 (2007).

10. Foletti, S., Bluhm, H., Mahalu, D., Umansky, V. \& Yacoby, A. Universal quantum control in two-electron spin quantum bits using dynamic nuclear polarization. Nat. Phys. 5, 903-908 (2009).

11. Bluhm, H., Foletti, S., Mahalu, D., Umansky, V. \& Yacoby, A. Enhancing the coherence of a spin qubit by operating it as a feedback loop that controls its nuclear spin bath. Phys. Rev. Lett. 105, 216803 (2010).

12. Bluhm, H. et al. Dephasing time of GaAs electron-spin qubits coupled to a nuclear bath exceeding $200 \mu$ s. Nat. Phys. 7, 109-113 (2011).

13. Sergeevich, A., Chandran, A., Combes, J., Bartlett, S. D. \& Wiseman, H. M. Characterization of a qubit Hamiltonian using adaptive measurements in a fixed basis. Phys. Rev. A 84, 052315 (2011).

14. Kestner, J. P., Wang, X., Bishop, L. S., Barnes, E. \& Das Sarma, S. Noiseresistant control for a spin qubit array. Phys. Rev. Lett. 110, 140502 (2013).

15. Coish, W. A. \& Loss, D. Hyperfine interaction in a quantum dot: nonMarkovian electron spin dynamics. Phys. Rev. B 70, 195340 (2004).

16. Klauser, D., Coish, W. A. \& Loss, D. Nuclear spin state narrowing via gatecontrolled Rabi oscillations in a double quantum dot. Phys. Rev. B 73, 205302 (2006).

17. Ferrie, C., Granade, C. E. \& Cory, D. G. How to best sample a periodic probability distribution, or on the accuracy of Hamiltonian finding strategies. Quantum Inf. Process. 12, 611 (2013).

18. Schroer, M. D. \& Petta, J. R. Quantum dots: time to get the nukes out. Nat. Phys. 4, 516-518 (2008)

19. Balasubramanian, G. et al. Ultralong spin coherence time in isotopically engineered diamond. Nat. Mater. 8, 383-387 (2009).

20. Wild, A. et al. Few electron double quantum dot in an isotopically purified $28 \mathrm{Si}$ quantum wel. Appl. Phys. Lett. 100, 143110 (2012).

21. Muhonen, J. T. et al. Storing quantum information for 30 seconds in a nanoelectronic device. Preprint at http://arxiv.org/abs/1402.7140 (2014).

22. Hahn, E. L. Spin echoes. Phys. Rev. 80, 580-594 (1950).

23. Uhrig, G. S. Exact results on dynamical decoupling by pi pulses in quantum inforamtion processes. New J. Phys. 10, 083024 (2008)
24. Nielsen, M. A. \& Chuang, I. L. Quantum Computation and Quantum Information (Cambridge Univ. Press, 2000).

25. Reed, M. D. et al. Realization of a three-qubit quantum error correction with superconducting circuits. Nature 482, 382-385 (2011).

26. Waldherr, G. et al. Quantum error correction in a solid-state hybrid spin register. Nature 506, 204-207 (2014).

27. Taminiau, T. H., Cramer, J., Van der Sar, T., Dobrovitski, V. V. \& Hanson, R. Universal control and error correction in multi-qubit spin registers in diamond. Nat. Nanotechnol. 9, 171-176 (2014).

\section{Acknowledgements}

We acknowledge James MacArthur for building the correlated double sampler. This research was funded by the United States Department of Defense, the Office of the Director of National Intelligence (ODNI), Intelligence Advanced Research Projects Activity (IARPA), and the Army Research Office grant (W911NF-11-1-0068 and W911NF-11-1-0068). All statements of fact, opinion or conclusions contained herein are those of the authors and should not be construed as representing the official views or policies either expressly or implied of of IARPA, the ODNI, or the U.S. Government. S.P.H was supported by the Department of Defense (DoD) through the National Defense Science \& Engineering Graduate Fellowship (NDSEG) Program. A.C.D. acknowledges discussions with Matthew Wadrop regarding extracting diffusion constants. A.C.D. and S.D.B. acknowledge support from the ARC via the Centre of Excellence in Engineering Quantum Systems (EQuS) project number CE110001013. This work was performed in part at the Center for Nanoscale Systems (CNS), a member of the National Nanotechnology Infrastructure Network (NNIN), which is supported by the National Science Foundation under NSF award no. ECS-0335765. CNS is a part of Harvard University.

\section{Author contributions}

V.U. prepared the crystal M.D.S. fabricated the sample, J.M.N. programmed the FPGA, M.D.S, S.P.H., J.M.N., S.D.B, A.C.D, and A.Y. carried out the experiment, analyzed the data, and wrote the paper.

\section{Additional information}

Supplementary Information accompanies this paper at http://www.nature.com/ naturecommunications

Competing financial interests: The authors declare no conflict of interest.

Reprints and permission information is available online at http://npg.nature.com/ reprintsandpermissions/

How to cite this article: Shulman, M. D. et al. Suppressing qubit dephasing using real-time Hamiltonian estimation. Nat. Commun. 5:5156 doi: 10.1038/ncomms6156 (2014)

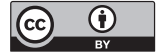

This work is licensed under a Creative Commons Attribution 4.0 International License. The images or other third party material in this article are included in the article's Creative Commons license, unless indicated otherwise in the credit line; if the material is not included under the Creative Commons license, users will need to obtain permission from the license holder to reproduce the material. To view a copy of this license, visit http://creativecommons.org/licenses/by/4.0/ 